\title{
Article
}

\section{Anti-inflammatory activities of hepatocyte growth factor in post-ischemic heart failure}

\author{
Shu-ling RONG ${ }^{1}$, Xiao-lin WANG ${ }^{2, *}$, Yi-cheng WANG ${ }^{3}$, Huan $W^{4}{ }^{4}$, Xue-dong ZHOU ${ }^{5}$, Ze-kun WANG ${ }^{5}$, Yu-chuan WANG ${ }^{1}$, Cun- \\ shui $\mathrm{XUE}^{6}$, Bao $\mathrm{LI}^{1, *}$, Dong-lai GAO ${ }^{1}$ \\ ${ }^{1}$ Department of Cardiology, The Second Hospital of Shanxi Medical University, Taiyuan 030001, China; ${ }^{2}$ Department of Pediatrics, \\ The Second Hospital of Shanxi Medical University, Taiyuan 030001, China; ${ }^{3}$ Department of Cardiology, Heping Hospital, Changzhi \\ Medical College, Changzhi 046000, China; ${ }^{4}$ Department of Pediatrics, Heping Hospital, Changzhi Medical College, Changzhi 046000, \\ China; ${ }^{5}$ Department of Conservative Dentistry, West China School of Stomatology and State Key Laboratory of Oral Diseases, Sichuan \\ University, Chengdu 610041, China; ${ }^{6}$ Department of Neurology, The Second Hospital of Shanxi Medical University, Taiyuan 030001, \\ China
}

\begin{abstract}
Hepatocyte growth factor (HGF) alleviates acute and chronic inflammation in experimental inflammatory bowel disease, glomerulonephritis, and airway inflammation. However, the anti-inflammatory effects of HGF on myocardial infarction are not defined. The current study assessed the anti-inflammatory effects of HGF in post-ischemic heart failure. The left anterior descending coronary artery was ligated in rats, and adenovirus containing human HGF (Ad-HGF) or control virus (Ad-GFP) was administered intramyocardially. The quantity of proinflammatory cytokines secreted by cardiomyocytes, such as tumor necrosis factor- $\alpha$ (TNF- $\alpha$ ), interleukin-6 (IL-6), and IL-1 $\beta$, was evaluated. Cardiac function and LV remodeling were assessed using echocardiography and collagen deposition, respectively. Left ventricular fractional shortening (LVFS) and left ventricular ejection fraction (LVEF) four weeks after injection were significantly increased in Ad-HGF-treated animals compared to the Ad-GFP group. HGF gene therapy improved ventricular geometry with a significantly decreased left ventricular end-diastolic diameter (LVEDD) and markedly reduced myocardial collagen deposition. Treatment with Ad-HGF significantly decreased the mRNA levels of TNF- $\alpha$, IL- 6 , and IL-1 $\beta$ in the non-infarcted region four weeks after injection. Changes of the TNF- $\alpha$, IL-6, and IL-1 $\beta$ levels in the non-infarcted region positively correlated with the LVEDD 4 weeks after infarction. Treatment of acute myocardial infarction (AMI) with Ad-HGF in the early stage of MI reduced the pro-inflammatory cytokine levels and preserved cardiac function. These findings indicated that Ad-HGF gene therapy alleviated ventricular remodeling after infarction by reducing inflammation.
\end{abstract}

Keywords: hepatocyte growth factor; gene therapy; ventricular remodeling; inflammatory cytokines; myocardial infarct

Acta Pharmacologica Sinica (2018) 39: 1613-1621; doi: 10.1038/aps.2018.14; published online 24 May 2018

\section{Introduction}

Acute myocardial ischemia (AMI) remains the major cause of heart failure and may cause death in humans. A large area of MI may cause multiple progressive structural, geometric, and functional alterations in the left ventricle (LV), termed ventricular remodeling, which ultimately lead to heart failure. Animal and human studies demonstrated that inflammatory cytokines, including tumor necrosis factor- $\alpha$ (TNF- $\alpha$ ), interleukin-6 (IL-6) and IL-1 $\beta$, play critical roles in the pathogenesis of $\mathrm{LV}$ remodeling following $\mathrm{AMI}^{[1-3]}$ and control heart structure

\footnotetext{
* To whom correspondence should be addressed.

E-mail czsrs।@sina.com (Xiao-lin WANG); libaoxys@163.com (Bao LI)

Received 2017-10-19 Accepted 2018-02-28
}

and function.

Hepatocyte growth factor (HGF) alleviates chronic myocardial injury in multiple models, such as AMI, myocarditis and cardiomyopathy. Mounting evidence reveals that HGF therapy is beneficial for MI because of it exhibits angiogenic, mitogenic, morphogenic, anti-apoptotic and anti-fibrotic properties. HGF ameliorates inflammation in several disease animal models, including inflammatory bowel disease, glomerulonephritis, and airway inflammation. HGF also modulates essential inflammatory reactions involved in multiple ailments and organs ${ }^{[4-10]}$. Therefore, HGF may act as a common suppressor of proinflammatory processes.

The role of the anti-inflammatory effects of HGF gene therapy in myocardial infarction remains largely unknown. We hypothesized that the beneficial effects of HGF gene therapy 
partially depend on the regulation of myocardial inflammation in the early stage of LV remodeling. The current study examined the impact of HGF on MI and early ventricular remodeling in a rat model of AMI.

\section{Materials and methods Animal handling}

Male Wistar rats were obtained from the Academy of Military Medical Science (Beijing, China) and maintained under conventional clean conditions with a 12 h/12 h light-dark cycle and standard rodent diet and water freely available. The animal experiments were performed with approval from the Institutional Animal Care and Use Committee of Shanxi Medical College.

\section{Adenoviral vectors}

HGF cDNA harboring (Ad-HGF) and control (Ad-GFP) adenoviruses were kindly provided by the Beijing Institute of Radiation Medicine. Adenovirus amplification was performed in HEK293 cells, and the infectious titers of the adenoviruses were determined as previously described ${ }^{[11]}$.

\section{Animal surgery}

MI was generated in male Wistar rats (220 to $260 \mathrm{~g}$ ) via left coronary ligation as described previously ${ }^{[11]}$. All animals in the treatment group (HGF group, $n=18$ ) received $1 \times 10^{10} \mathrm{pfu}$ Ad-HGF adenovirus intramyocardially, and a thoracotomy ligation was performed using a $30-\mathrm{G}$ needle. The adenovirus suspension $(200 \mu \mathrm{L})$ was administered at four different sites. Control rats (GFP group, $n=18$ ) received comparable injections of Ad-GFP intramyocardially, and a thoracotomy ligation was performed using a 30-G needle. Thirty-two rats were ligated and randomly assigned to the HGF $(n=16)$ or GFP $(n=16)$ groups and assessed 7 days after MI. Sham-operated rats underwent the same procedure without coronary artery ligation.

\section{Echocardiographic analysis}

Two-dimensional echocardiography was performed on each rat 4 weeks after treatment with a $10-\mathrm{MHz}$ (short focus) transducer (GE Vivid 7, USA). LV-end-diastolic diameter (LVEDD), fractional shortening (LVFS), and ejection fraction (LVEF) were determined.

Assessment of collagen deposition, vessel density, and apoptosis The heart was arrested in the diastolic phase after echocardiography via intravenous injection of $2 \pm 3 \mathrm{~mL} 10 \% \mathrm{KCl}$. The extracted hearts were perfused with phosphate-buffered saline (PBS) and snap frozen. Transverse sections (2 mm) of left ventricle samples were fixed with $10 \%$ formaldehyde, paraffinembedded, and serially sectioned at $4 \mu \mathrm{m}$. Hematoxylin and eosin (H\&E) staining was performed, and images were acquired. The LV free wall and infarct size were evaluated using Scion Image software. The infarct size was determined as a percentage of the infarct scar based on the LV free wall. The collagen-specific stain Sirius Red was used, and noninfarcted myocardial areas were assessed after imaging. Inter- stitial fibrosis areas were assessed relative to the total connective tissue area.

Vascular density and cardiomyocyte apoptosis in noninfarcted areas were assessed 4 weeks after treatment. Antibodies targeting factor VIII and a-smooth muscle actin (a-SMA) (Zhongshan Reagent Company, Beijing) were used to immunohistochemically quantitate the vascular density in the border zone. Two investigators quantified a-SMA+ and factor VIII+ arterioles in 5 high power fields per sample in a blinded manner.

Cardiomyocyte apoptosis was evaluated using TUNEL staining with an In Situ Cell Death Detection Kit (Roche, Germany) as directed by the manufacturer 4 weeks after treatment. TUNEL-positive myocytes in non-infarcted areas were quantified in 10 section. The percentage of apoptotic nuclei was measured in 6 randomly selected fields.

\section{Determination of the inflammatory cytokine expression levels using immunohistochemistry}

Anti-rat TNF-a, IL-6, and IL-1 (1:200) antibodies were incubated with tissue sections overnight at $4{ }^{\circ} \mathrm{C}$. Sections were incubated with biotinylated secondary antibodies at room temperature for $30 \mathrm{~min}$. Immunoreactivity was assessed using microscopy and HPIAS-2000 software.

\section{Assessment of gene expression using quantitative RT-PCR (qRT- PCR)}

Total RNA from non-infarcted myocardium was extracted using TRIzol reagent (Invitrogen, USA) and submitted to reverse transcription using a RevertAid First Strand cDNA Synthesis Kit (Thermo Scientific, Wilmington, DE, USA) following the manufacturer's instructions. Cytokine and GAPDH mRNA levels were assessed using qRT-PCR. The sense primer and the antisense primer in rat for TNF$\alpha$, IL-6, IL-1 $\beta$ and GAPDH were as follows: TNF-a (691 bp): 5' -ATGAGCACGGAAAGCATGATCCGA-3'; and 5'-CCAAAGTAGACCTGCCCGGACTC-3'; IL-6 (295 bp): 5'-AGAGCAATACTGAAACCCTA-3'; and 5'-CAACATAATTTAGATACCCATC-3'; IL-1 $\beta$ (555 bp): 5'-ATGGCAACTGTCCCTGAACTCAACT-3'; and 5'-CAGGACAGGTATAGATTCAACCCCT-3'; GAPDH (213 bp): 5'-TATGATGACATCAAGAAGGTGG-3'; and 5'-CACCACCCTGTTGCTGTA-3'. Amplicons were resolved using 1.5\% agarose gel electrophoresis and were quantified using a digital image analysis system (GSD8000, UVP, UK). GAPDH was used for normalization.

\section{Determination of protein levels using immunoblotting}

Total protein was obtained from non-infarcted areas of the left heart and quantified using a Coomassie brilliant blue G250 assay kit. Equal amounts of protein were resolved using 10\% SDS-PAGE, and separated proteins were electrotransferred onto nitrocellulose membranes. Membranes were blocked with 5\% non-fat milk in TBS and successively incubated with primary antibodies (polyclonal goat anti-rat TNF-a or IL-6 [R\&D], monoclonal mouse anti-rat IL-1 $\beta$ (Serotec), polyclonal rabbit anti-rat bcl-2, bax, or caspase-3 (Santa Cruz) at $4{ }^{\circ} \mathrm{C}$ 
overnight and then the appropriate horseradish peroxidaseconjugated secondary antibodies at $37^{\circ} \mathrm{C}$ for $1 \mathrm{~h}$. Immunoreactive bands were visualized using the Chemiluminescent Detection System. Semi-quantitative analysis was performed using densitometry.

\section{Statistical analysis}

Data are presented as the means \pm SD. Statistical analysis of the data was performed with one-way ANOVA (more than two groups) using SPSS20.0, and $P<0.05$ indicated statistical significance.

\section{Results}

\section{Successful HGF overexpression}

GFP expression in rat heart was detected in frozen left ventricular samples one week after Ad-HGF and Ad-GFP adenovirus administration using fluorescence microscopy (Figure 1A). HGF expression in the treated heart was detected using Western blotting. Figure 1B shows marked overexpression of hHGF in the Ad-HGF treatment group 4 weeks post-treatment.

\section{Cardiac function}

Echocardiography was performed to detect cardiac functions 4 weeks after treatment. Compared with the sham group, there were significantly increased LVEDD (Figure $2 \mathrm{~A}, P<0.05$ ), markedly decreased LVFS and LVEF (Figure 2B, 2C, both $P<0.05)$ in the GFP group. Ad-HGF treatment significantly attenuated LV dilatation and markedly improved LV function compared to the Ad-GFP group.

\section{Myocardial morphometric features, infarct size, and lymphocyte counting}

MI rats were treated with Ad-GFP and Ad-HGF for 4 weeks. Hearts in the GFP group (Figure 3A) exhibited significant LV dilatation compared to the sham group (Figure 3C), and HGF treatment (Figure 3B) reduced the LV cavity. The infract size increased significantly and the left ventricular free wall thickness markedly decreased in the GFP group according to histological analyses. HGF markedly reduced the infarct size and increased the wall thicknesses (Figure 3D, 3E, $P<0.05$ ).

Histological differences between MI rats treated with AdHGF or Ad-GFP were further investigated. The histological changes included myocardial fiber fracture and infiltration of large numbers of inflammatory cells (Figure 3F, 3G and 3H). The number of lymphocytes increased significantly in the infarcted area in the GFP group compared to the sham group. Ad-HGF treatment significantly protected myocardiocytes and reduced inflammatory cell infiltration (Figure 3I, $P<0.05$ ).

Collagen deposition and vascular density in non-infarcted myocardial areas

Collagen deposition in infarcted and non-infarcted areas was assessed using quantitative imaging 4 weeks after MI (Figure $4 \mathrm{~A}, 4 \mathrm{~B}, 4 \mathrm{C}, 4 \mathrm{E}, 4 \mathrm{~F}$, and $4 \mathrm{G}$ ). Ad-HGF treatment significantly attenuated collagen deposition in AMI rats compared to the Ad-GFP group (Figure 4D and $4 \mathrm{H}, P<0.05$ ).
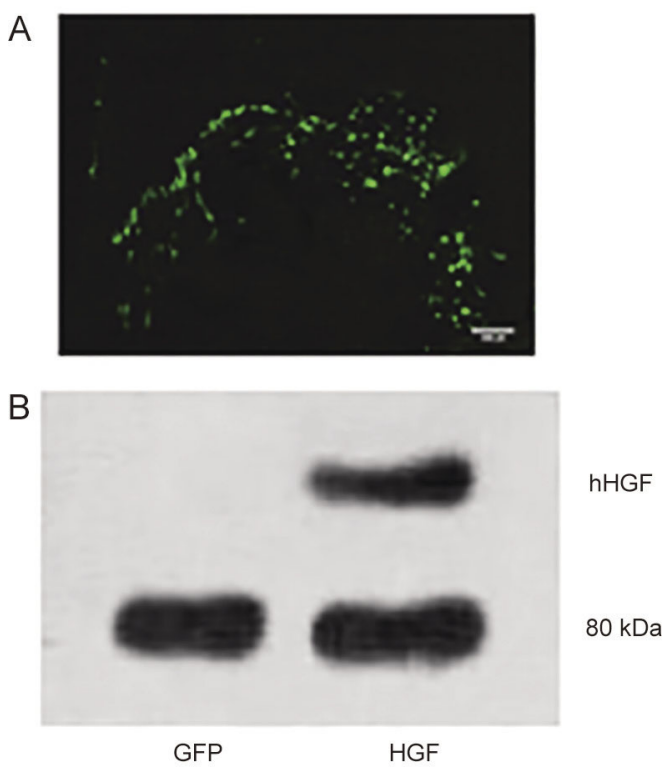

Figure 1. (A) GFP expression in rat heart 1 week after direct Ad-GFP administration. Green fluorescence reveals GFP expression throughout the injection area in the left ventricular free wall. (B) Immunoblotting of HGF protein levels 1 week after infarction and adenovirus delivery.

New vessels (Factor $\mathrm{VIII}^{+}$and a-SMA ${ }^{+}$) were detected immunohistochemically in the non-infarcted myocardium in all groups 4 weeks after MI (Figure 4I, 4J, 4K, 4M, 4N, 4O). Vascular density increased significantly in the HGF group compared to the GFP group (Figure $4 \mathrm{~L}, 4 \mathrm{P}, P<0.05$ ).

\section{Apoptotic cells in the non-infarcted myocardium}

Cardiomyocyte apoptosis in non-infarcted myocardium was evaluated using TUNEL staining 4 weeks after MI (Figure 5A, 5B, 5C). Starkly fewer TUNEL-positive nuclei were observed in the HGF group compared to the GFP group (Figure $5 \mathrm{D}, P<0.05$ ). Immunoblots (Figure 5E) revealed that markedly reduced bax and cleaved caspase- 3 protein levels and increased bcl-2 levels in non-infarcted areas in the HGF group than the GFP group (Figure 5F, 5G, 5H, P<0.05).

\section{Ad-HGF treatment reduces myocardial expression levels of inflammatory cytokines}

Heart samples were assessed immunohistochemically for TNF-a (Figure 6A-C), IL-6 (Figure 6E-G), and IL-1 $\beta 4$ weeks after treatment (Figure 6I-K). Cytokines were primarily found in the non-infarct area. Ad-HGF treatment significantly decreased TNF- $\alpha$ (Figure 6D), IL-6 (Figure 6H) and IL-1 $\beta$ (Figure 6L) protein levels (all $P<0.05$ ).

qRT-PCR demonstrated that gene expression levels of TNF- $\alpha$, IL-6, and IL-1 $\beta$ were markedly elevated in noninfarcted regions 4 weeks post-surgery. The HGF group exhibited significantly reduced TNF- $\alpha$, IL- 6 , and IL-1 $\beta$ mRNA levels compared to the GFP group (Figure $7, P<0.05$ ).

Immunoblot revealed that TNF- $\alpha$, IL- 6 , and IL-1 $\beta$ protein levels were markedly higher in non-infarcted regions 4 weeks 
A

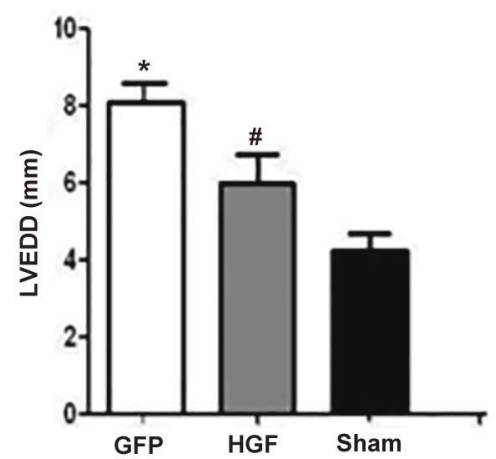

B

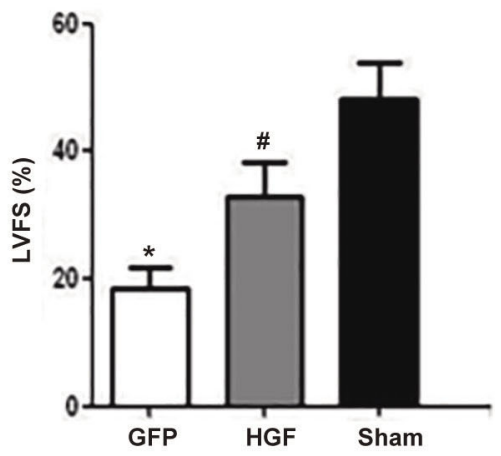

$\mathrm{C}$

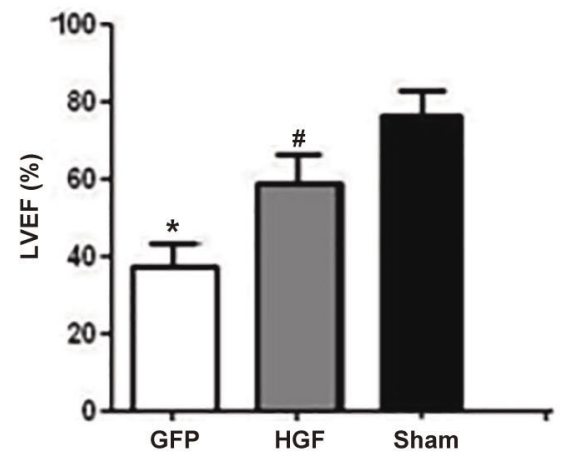

Figure 2. Cardiac function in Ad-HGF- and Ad-GFP-treated MI rats. MI rats were treated with Ad-HGF and Ad-GFP for 4 weeks, and LVEDD (A), LVFS (B), and LVEF (C) were detected using echocardiography. Values are mean \pm SD. $n=8$ /group. ${ }^{*} P<0.05$ vs sham group. ${ }^{*} P<0.05$ vs GFP group.

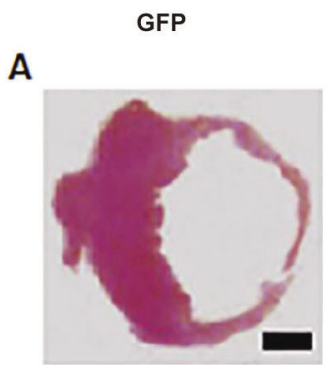

F

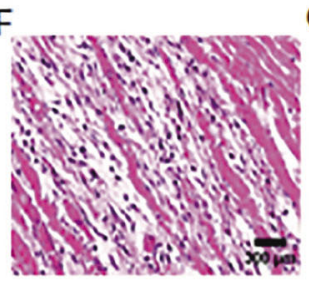

HGF

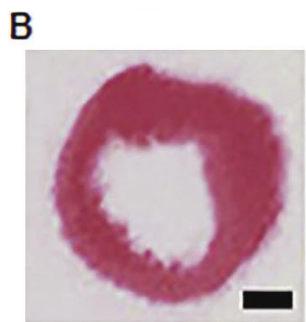

G

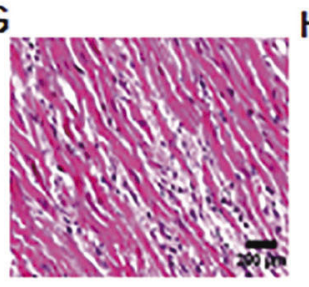

C

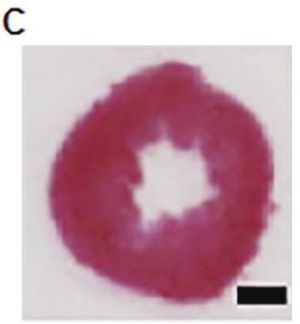

$\mathrm{H}$

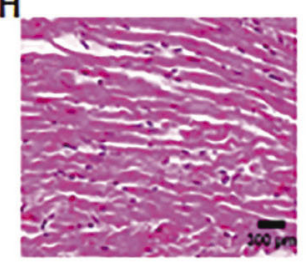

D

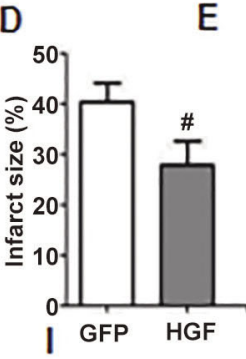

E
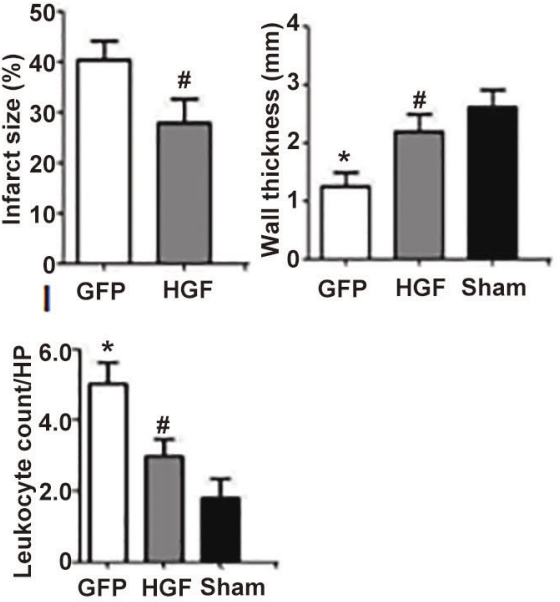

Figure 3. Cardiac morphology and hematoxylin and eosin staining 4 weeks after treatment. Cardiac morphological changes in the GFP group (A), HGF group (B) and sham group (C) (H\&E staining, Scale bar=1 mm). HGF markedly reduced the infarct size (D) and increased the wall thickness (E). Representative photomicrographs of $\mathrm{H} \& \mathrm{E}$ staining $(\mathrm{F}, \mathrm{G}$ and $\mathrm{H} ; \times 100)$. The number of infiltrated lymphocytes significantly decreased in the HGF group compared to the Ad-GFP group (I). Values are mean \pm SD. $n=8$ /group. ${ }^{*} P<0.05$ vs sham group. ${ }^{\#} P<0.05$ vs GFP group.

post-surgery. HGF treatment markedly decreased TNF-a, IL-6, and IL-1 $\beta$ protein levels (Figure $8, P<0.05$ ).

\section{Associations of LVEDD with cytokine gene expression levels}

We analyzed the associations of LVEDD with cytokine mRNA expression levels. Increases in cytokine expression levels in the non-infarcted heart were correlated with LVEDD 4 weeks after surgery (Figure 9).

\section{Discussion}

The present study demonstrated that HGF gene transfection improved LV remodeling in rats with AMI. This effect was associated with the induction of angiogenesis, suppression of cardiomyocyte apoptosis, and decreased pro-inflammatory cytokine expression.

The anti-inflammatory effects contributed to the therapeutic properties of Ad-HGF in AMI. First, Ad-HGF treatment in the early stage of AMI attenuated LV remodeling. LV dilation was obvious 4 weeks post-AMI in our rat model. Reduced factional shortening and ejection fraction and elevated collagen I synthesis were observed in non-infarcted areas. Ad-HGF treatment increased factional shortening and ejection fraction and decreased collagen deposition. Ad-HGF treatment in early stage of AMI decreased the gene expression levels of pro-inflammatory cytokines in non-infarcted areas. Cytokine expression changes were tightly associated with improve- 


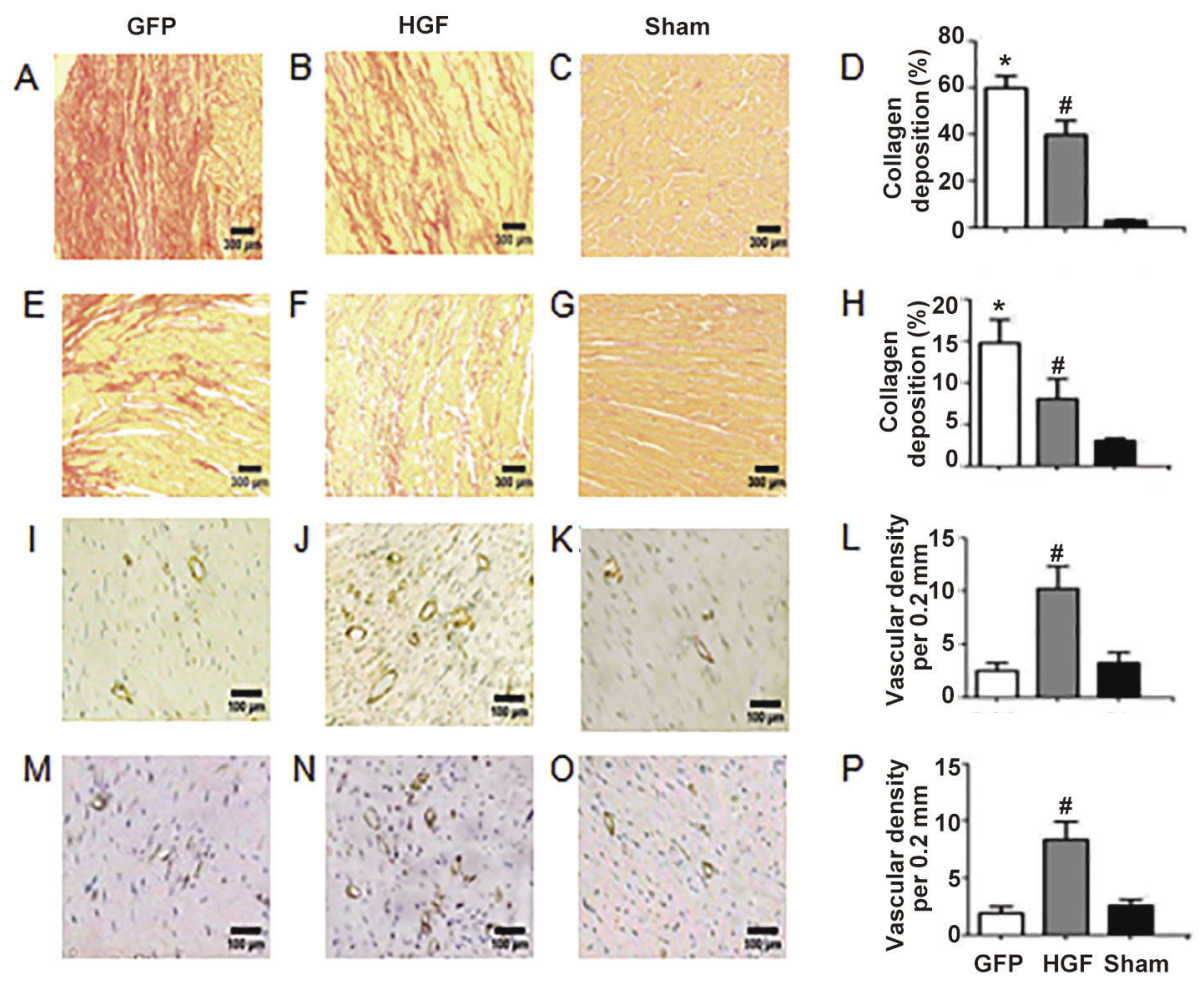

Figure 4. Effects of Ad-HGF treatment on collagen deposition and vascular density in the myocardium 4 weeks after treatment. Collagen deposition was assessed using Sirius red staining in cardiac sections from the GFP group ( $A$ and $E)$, HGF group $(B$ and $F)$ and sham group $(C$ and $G)(\times 100)$. The graph shows the percent areas of collagen fiber in infarct myocardium (D) and non-infarct myocardium (H). Values are mean \pm SD. $n=8 /$ group. ${ }^{*} P<0.05$ vs sham group. ${ }^{\#} P<0.05$ vs GFP group. Representative photomicrographs for capillary density assessment in non-infarct areas. Sections were stained with antiFactor VIII (I-K) and $\alpha$-SMA antibodies $(M-0)$ to assess vessel numbers $(\times 400)$. Vascular density increased significantly in the HGF group compared to the GFP group ( $L$ and $P$ ). Values are mean \pm SD. $n=8$ /group. ${ }^{*} P<0.05$ vs sham group. ${ }^{\#} P<0.05$ vs GFP group.
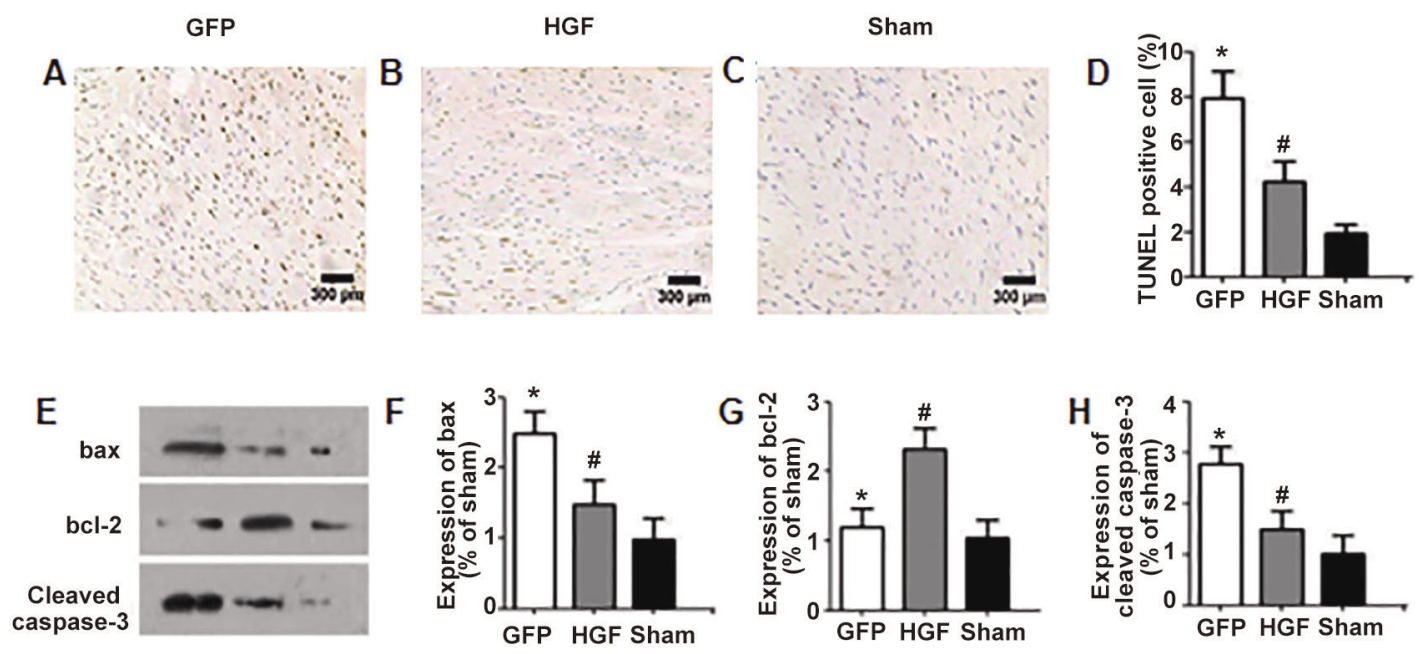

Figure 5. Effect of HGF on myocyte apoptosis 4 weeks after treatment. Effects of Ad-HGF treatment on myocyte apoptosis in the GFP group (A), HGF group (B) and sham group (C) $(\times 100)$. The percentages of TUNEL-positive myocytes in the non-infarcted areas (D). Immunoblot assessment of bax, bcl-2 and cleaved caspase-3 protein levels in the left ventricular myocardium (E). Graph of average relative expression of bax, bcl-2 and cleaved caspase-3 protein in each experimental group $(\mathrm{F}, \mathrm{G}, \mathrm{H})$. Values are mean \pm SD. $n=8$ /group. ${ }^{*} P<0.05$ vs sham group. ${ }^{\#} P<0.05$ vs GFP group. 

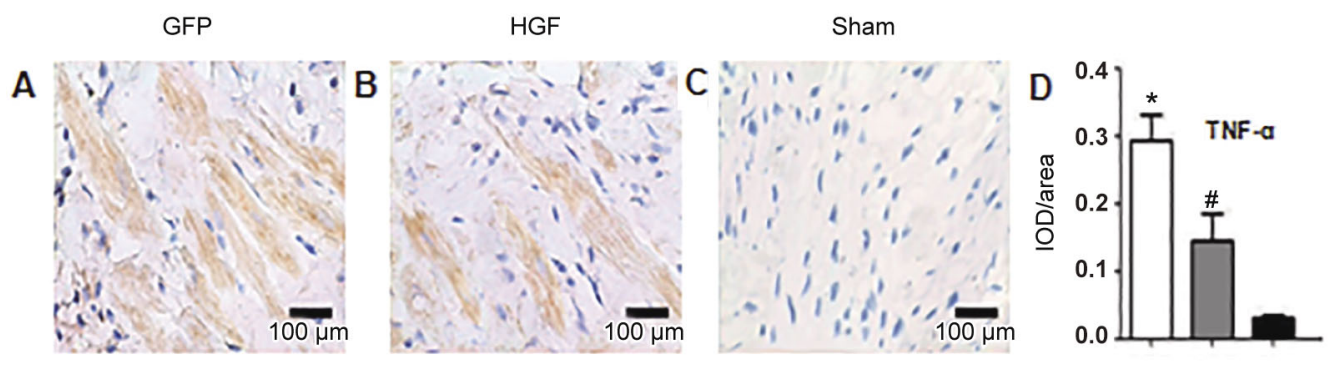

$\mathrm{E}$
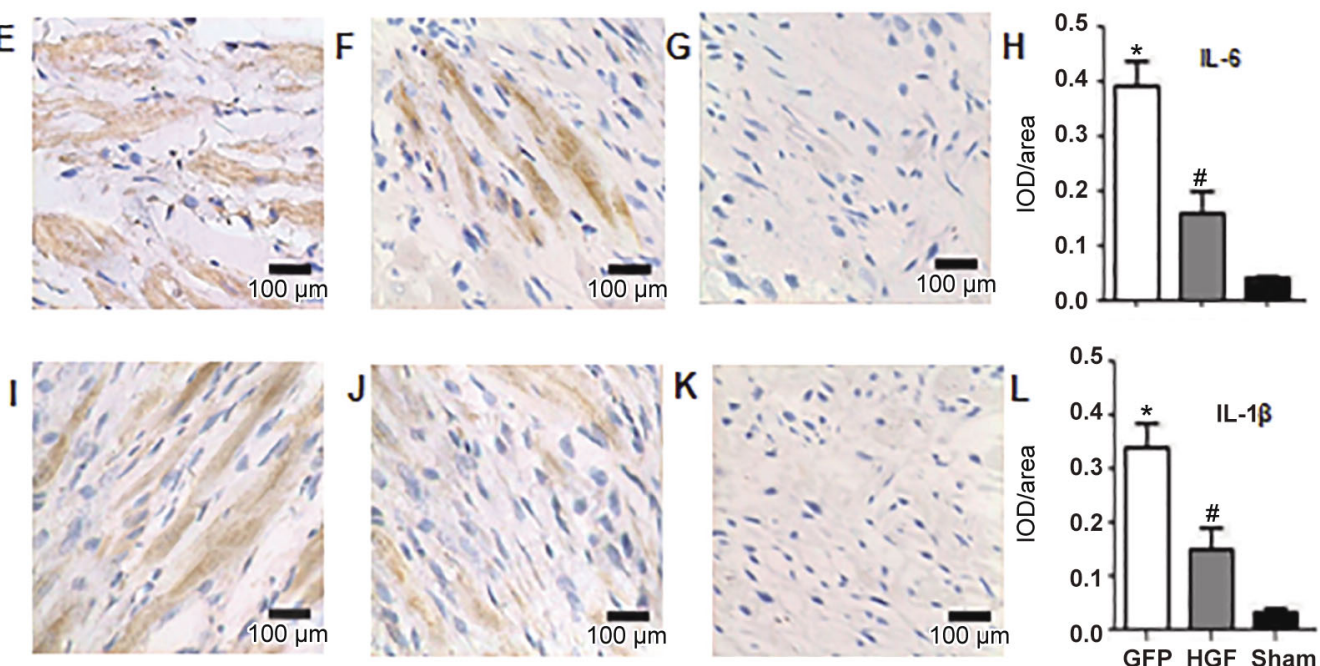

Figure 6. Representative micrographs showing the localization of TNF- $\alpha(A-C)$, IL-6 $(E-G)$ and IL-1 $1 /(\mathrm{I}-\mathrm{K})$ in the non-infarcted myocardium as assessed using immunohistochemistry ( $\times 400$ ). IOD/area semiquantitation of TNF- $\alpha(D), I L-6(H)$ and IL-1 $\beta$ (L) show marked differences between the GFP group, HGF group and sham group. Values are mean \pm SD. $n=8$ /group. ${ }^{*} P<0.05$ vs sham group. ${ }^{\#} P<0.05$ vs GFP group.
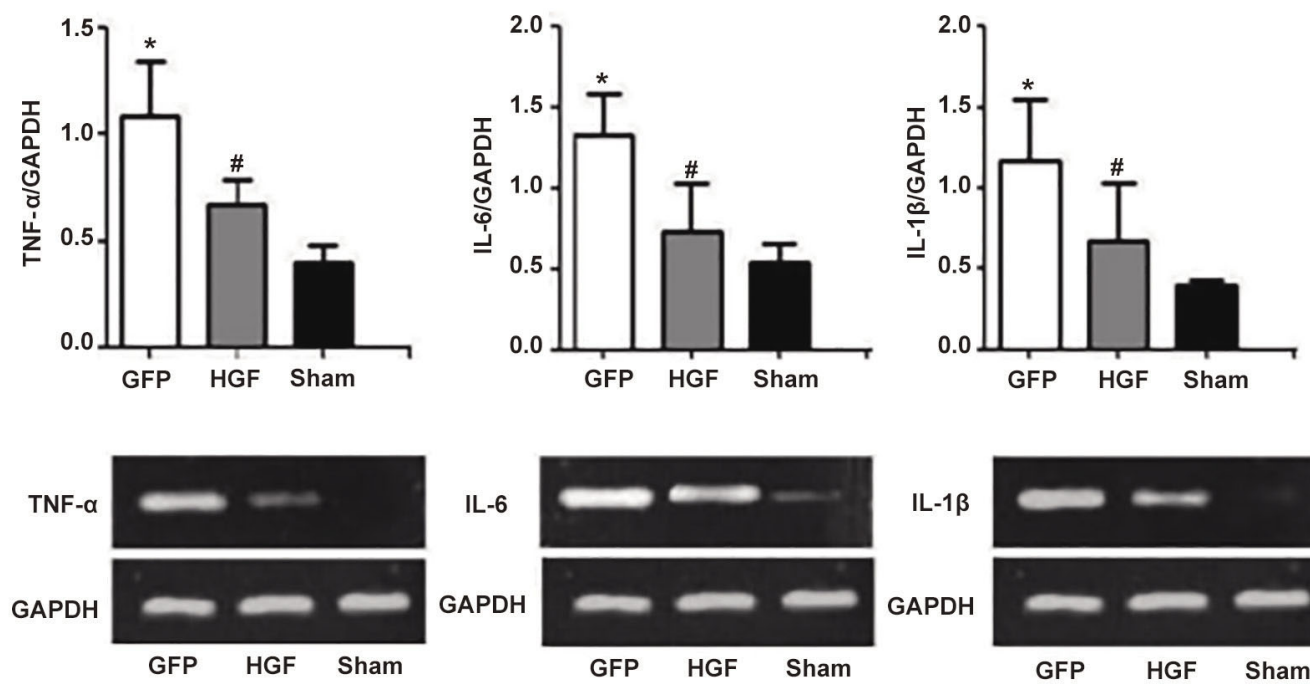

IL-1 $1 \beta$

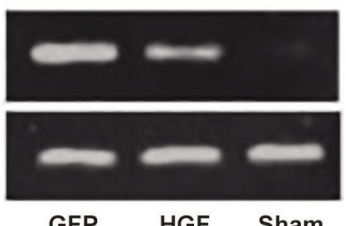

Figure 7. Effects of HGF on mRNA levels of inflammatory cytokines in non-infarcted areas of the LV myocardium 4 weeks after treatment. Values are mean \pm SD. $n=8$ /group. ${ }^{*} P<0.05$ vs sham group. ${ }^{\#} P<0.05$ vs GFP group. 


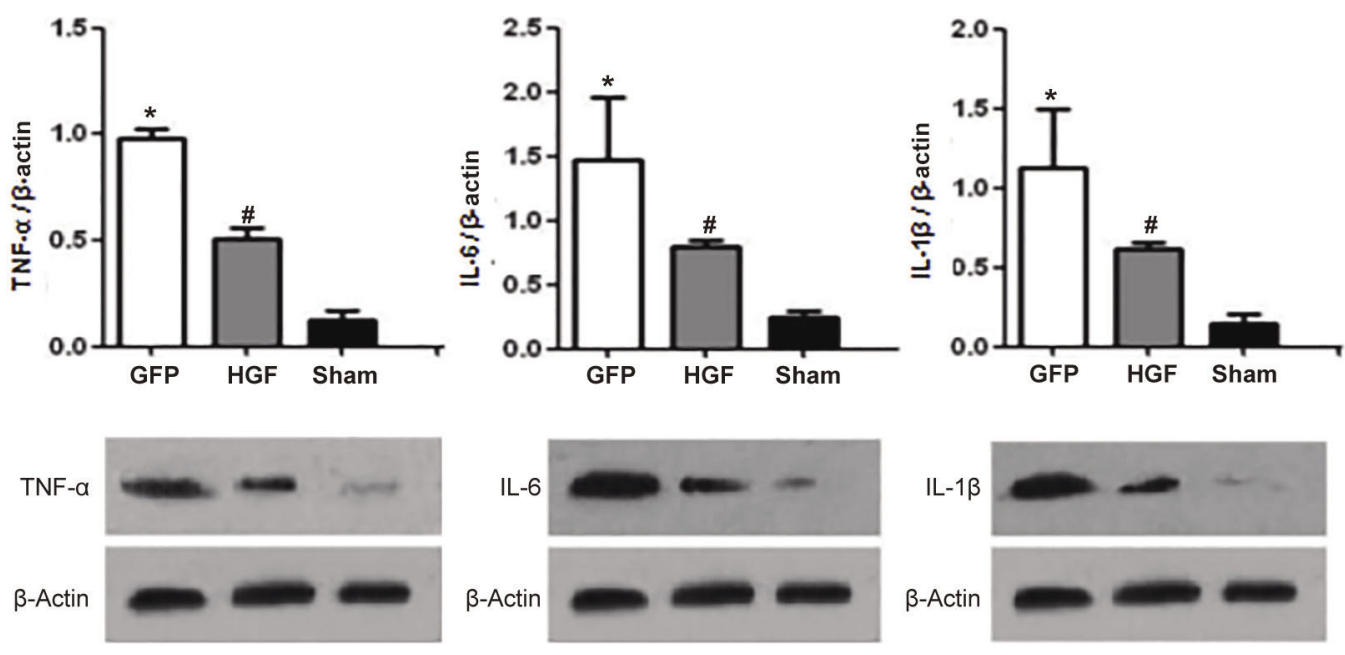

Figure 8. Effects of HGF on cytokine protein levels in non-infarcted areas of the LV myocardium 4 weeks after treatment. Values are mean \pm SD. $n=8 /$ group; ${ }^{*} P<0.05$ vs sham group. ${ }^{\#} P<0.05$ vs GFP group.
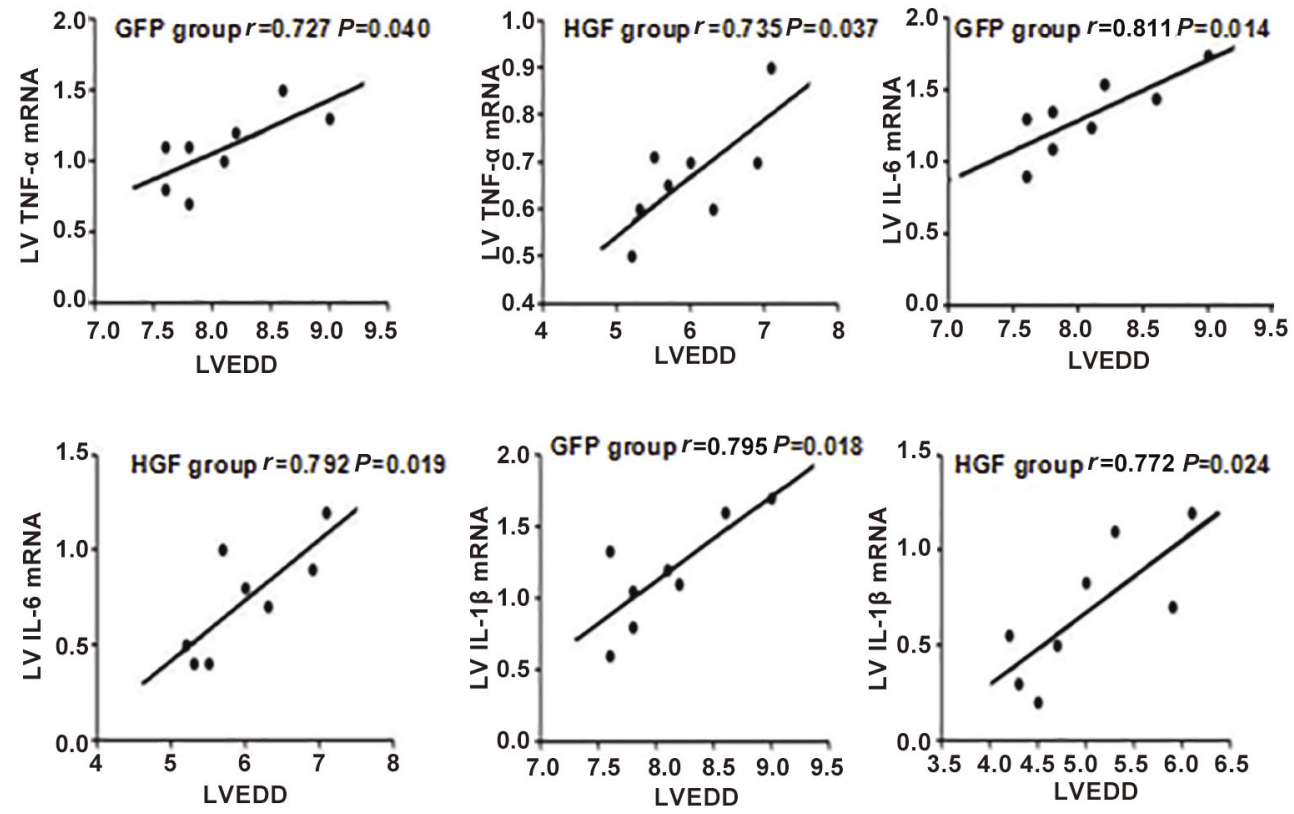

Figure 9. Associations of LVEDD with TNF- $\alpha$, IL-6, and IL-1 $1 \beta$ gene expression levels in the non-infarcted area 4 weeks after treatment. The expression levels of each cytokine were positively correlated with LVEDD.

ments in cardiac function. These findings suggest that HGF improves early ventricular remodeling after MI in animals, likely via inflammation modulation.

\section{Inflammatory cytokines and post-infarct LV dysfunction}

Ventricular remodeling is the primary reason for post-MI heart failure, and it is characterized by ventricular dilatation and reduced cardiac function ${ }^{[3,12-15]}$. Cardiac remodeling after a pronounced MI includes hypertrophy of the surviving myo- cytes and non-myocyte hyperplasia. Multiple inflammatory cytokines are upregulated during this process, and higher levels are observed in the circulation and cardiomyocytes ${ }^{[15-18]}$. Cytokines include proinflammatory (eg, TNF- $\alpha$, IL-1 $\beta$ and IL-6) and anti-inflammatory (eg, IL-10) types ${ }^{[12]}$. An imbalance between pro- and anti-inflammatory cytokines is involved in ventricular remodeling after MI. Blood cytokine levels may be associated with myocardial ischemia and cardiac dysfunction, which may subsequently alter the growth of myocytes 
and non-myocytes. Ono et $a l^{[3]}$ demonstrated that rats with MI exhibited starkly elevated TNF- $\alpha$, IL-1 $\beta$, and IL- 6 mRNA levels in infarcted and non-infarcted areas. Cytokines regulation in the non-infarcted myocardium is not clear, but cytokine levels in the non-infarcted area were associated with LVEDD and collagen deposition in the latter report ${ }^{[3]}$, which indicates a role for these cytokines in the signaling pathways that control cardiac remodeling. Multiple cytokines control cardiac myocyte growth, contractile protein production, fibroblast proliferation, and extracellular matrix production ${ }^{[3.15 .16 .17 .18]}$. Zhou et al ${ }^{[18]}$ revealed that IL-17A enhanced metalloproteinase-2 (MMP-2) activity, which reflected its pro-fibrotic effect post-MI. Platis et al ${ }^{[17]}$ demonstrated that IL-18 was involved in extracellular matrix synthesis and myocardial fibrosis. IL-10 is an anti-inflammatory cytokine that downregulates MMPs and upregulates tissue inhibitors (TIMPs) to modulate extracellular matrix remodeling ${ }^{[12]}$.

These findings indicated that cytokines were critical regulators of post-myocardial infarction remodeling processes, such as infarct-related inflammation, cardiac hypertrophy, myocardial fibrosis, and reduced cardiac function. Significantly reduced TNF- $\alpha$, IL- 6 and IL- $1 \beta$ amounts were found, which at least partially reflected restoration of the altered inflammatory balance related to advanced congestive heart failure (CHF). Data from the current study also confirmed this hypothesis.

\section{Effect of HGF on ventricular function and ventricular remodeling after MI}

HGF plays a critical role in early heart development ${ }^{[19,20-23]}$. HGF expression is associated with multiple cell-protective mechanisms: it induces mitogenicity, inhibits cell apoptosis, enhances angiogenesis, and reduces myocardial fibrosis. Jayasankar et a ${ }^{[20]}$ used HGF gene therapy for post-infarction heart failure and demonstrated that HGF gene transfection decreased left ventricular dilatation and preserved the contractile function and wall thickness 6 weeks after pronounced myocardial infarction in a rat model. These effects likely resulted from the combined angiogenic and anti-apoptotic effects of HGF. Duan et al also demonstrated that HGF genemodified mesenchymal stem cells (MSCs) promoted neovascularization and recovery from myocyte atrophy and improved perfusion and heart function in MI rats. These therapeutic effects were attributable to the ability of HGF to attenuate fibrosis and increase angiogenesis. The current study corroborated these findings. Ad-HGF administration starkly improved $\mathrm{LV}$ remodeling in MI model rats, which was reflected in the elevated LVFS and LVEF and reduced LVEDD. Ad-HGF induced angiogenesis, decreased collagen deposition, and suppressed apoptosis, which primarily contribute to fibrosis and subsequent heart failure.

\section{Effects of HGF on inflammatory cytokine expression}

HGF reduces inflammation in multiple organs and disease models, such as myocarditis ${ }^{[24]}$, glomerulonephritis ${ }^{[25.26]}$, inflammatory bowel disease ${ }^{[6]}$, collagen-induced arthritis ${ }^{[27]}$, pulmonary fibrosis ${ }^{[28]}$, allogeneic heart transplantation graft-vs -host disease ${ }^{[29]}$, and asthma ${ }^{[10]}$. Whether the anti-inflammatory activity of HGF is involved in the therapeutic effects of AdHGF in MI is not known. This study demonstrated that AdHGF treatment decreased gene and protein expression levels of TNF- $\alpha$, IL-1 $\beta$, and IL-6 in rats with MI. These findings suggest that HGF ameliorates LV remodeling, at least partially via the downregulation of TNF- $\alpha$, IL- 6 , and IL- $1 \beta$. The mechanisms of HGF regulation of pro-inflammatory cytokines in MI models are not clear, but its role in the treatment of postinfarction heart failure was validated.

In conclusion, this study revealed that modulation of inflammatory cytokines may contribute to the therapeutic effects of HGF in MI. These results enhance our understanding of the pathogenesis and progression of ventricular remodeling and suggest that regulation of inflammatory cytokines may improve therapeutic outcomes in MI.

\section{Acknowledgements}

This work was supported by the National Natural Science Foundation of China (№ 81441014 to Bao LI), the Shanxi Province Natural Science Foundation (№ 2012011040-3 to Xiaolin WANG, 2013011049-4 to Shu-ling RONG), the Scientific Research Foundation of High Education Institutions of Shanxi Province (№ 200811034 to Shu-ling RONG), the Research Project of Shanxi Provincial Health and Family Planning Commission (201602026 to Shu-ling RONG, 201602028 to Xiao-lin WANG), the High School 131 Leading Talent Project of Shanxi Province to Shu-ling RONG, and the National Undergraduate Innovation and Entrepreneurship Training Programs (201610117001 to Shu-ling RONG).

\section{Author contribution}

Shu-ling RONG, Xiao-lin WANG and Bao LI conceived and designed the experiments; Shu-ling RONG, Xiao-lin WANG, Yi-cheng WANG, Huan WU, Ze-kun WANG and Yu-chuan WANG performed the research; Shu-ling RONG, Xiaolin WANG, Bao LI and Xue-dong ZHOU contributed new reagents or analytical tools; Xue-dong ZHOU, Cun-shui XUE and Shu-ling RONG analyzed the data; Shu-ling RONG, Xiaolin WANG, Ze-kun WANG, Yi-cheng WANG, and Dong-lai GAO wrote the manuscript.

\section{References}

1 Ma Y, Zou H, Zhu XX, Pang J, Xu Q, Jin QY, et al. Transforming growth factor $\beta$ : A potential biomarker and therapeutic target of ventricular remodeling. Oncotarget 2017; 8: 53780-90.

2 Nian M, Lee P, Khaper N, Liu P. Inflammatory cytokines and postmyocardial infarction remodeling. Circ Res 2004; 94: 1543-53.

3 Ono K, Matsumori A, Shioi T, Furukawa Y, Sasayama S. Cytokine gene expression after myocardial infarction in rat hearts: possible implication in left ventricular remodeling. Circulation 1998; 98: 14956.

4 Arthur LG, Kuenzler KA, Schwartz MZ. Hepatocyte growth factor ameliorates inflammatory bowel disease in a rat model. J Gastrointest Surg 2003; 7: 1062-8.

5 Arthur LG, Schwartz MZ, Kuenzler KA, Birbe R. Hepatocyte growth factor treatment ameliorates diarrhea and bowel inflammation in a rat model of inflammatory bowel disease. J Pediatr Surg 2004; 39: 
139-43.

6 Oh K, limuro Y, Takeuchi M, Kaneda Y, Iwasaki T, Terada N, et al. Ameliorating effect of hepatocyte growth factor on inflammatory bowel disease in a murine model. Am J Physiol Gastrointest Liver Physiol 2005; 288: G729-35.

7 Ohda Y, Hori K, Tomita T, Hida N, Kosaka T, Fukuda Y, et al. Effects of hepatocyte growth factor on rat inflammatory bowel disease models. Dig Dis Sci 2005; 50: 914-21.

8 Numata M, Ido A, Moriuchi A, Kim I, Tahara Y, Yamamoto S, et al. Hepatocyte growth factor facilitates the repair of large colonic ulcers in 2,4,6-trinitrobenzene sulfonic acid-induced colitis in rats. Inflamm Bowel Dis 2005; 11: 551-8.

9 Hanawa T, Suzuki K, Kawauchi Y, Takamura M, Yoneyama H, Han GD, et al. Attenuation of mouse acute colitis by naked hepatocyte growth factor gene transfer into the liver. J Gene Med 2006; 8: 623-35.

10 Ito W, Kanehiro A, Matsumoto K, Hirano A, Ono K, Maruyama H, et al. Hepatocyte growth factor attenuates airway hyperresponsiveness, inflammation, and remodeling. Am J Respir Cell Mol Biol 2005; 32 : 268-80.

11 Rong SL, Wang XL, Zhang CY, Song ZH, Cui LH, He XF, et al. Transplantation of HGF gene- engineered skeletal myoblasts improve infarction recovery in a rat myocardial ischemia model. PLoS One 2017; 12: e0175807.

12 Li B, Liao YH, Cheng X, Ge H, Guo H, Wang M. Effects of carvedilol on cardiac cytokines expression and remodeling in rat with acute myocardial infarction. Int J Cardiol 2006; 111: 247-55.

13 Xiao X, Chang G, Liu J, Sun G, Liu L, Qin S, et al. Simvastatin ameliorates ventricular remodeling via the TGF $\beta 1$ signaling pathway in rats following myocardial infarction. Mol Med Rep 2016; 13: 5093101.

14 Yang ZJ, Chen B, Sheng Z, Zhang DG, Jia EZ, Wang W, et al. Improvement of heart function in postinfarct heart failure swine models after hepatocyte growth factor gene transfer: comparison of low-, medium- and high-dose groups. Mol Biol Rep 2010; 37: 207581.

15 Sutton MG, Sharpe N. Left ventricular remodeling after myocardial infarction: pathophysiology and therapy. Circulation 2000; 101: 2981-8.

16 Konstam MA, Kramer DG, Patel AR, Maron MS, Udelson JE. Left ventricular remodeling in heart failure: current concepts in clinical significance and assessment. JACC Cardiovasc Imaging 2011; 4: 98-108.

17 Platis A, Yu Q, Moore D, Khojeini E, Tsau P, Larson D. The effect of daily administration of IL-18 on cardiac structure and function. Perfusion 2008; 23: 237-42.

18 Zhou SF, Yuan J, Liao MY, Xia N, Tang TT, Li JJ, et al. IL-17A promotes ventricular remodeling after myocardial. J Mol Med (Berl) 2014; 92: 1105-16.

19 Nakamura T, Sakai K, Nakamura T, Matsumoto K. Hepatocyte growth factor twenty years on: Much more than a growth factor. J Gastroenterol Hepatol 2011; 26 Suppl 1: 188-202.

20 Jayasankar V, Woo YJ, Bish LT, Pirolli TJ, Chatterjee S, Berry MF, et al. Gene transfer of hepatocyte growth factor attenuates postinfarction heart failure. Circulation 2003; 108 Suppl 1: II230-6.

21 Duan HF, Wu CT, Wu DL, Lu Y, Liu HJ, Ha XQ, et al. Treatment of myocardial ischemia with bone marrow-derived mesenchymal stem cells overexpressing hepatocyte growth factor. Mol Ther 2003; 8: 467-74.

22 Madonna R, Cevik C, Nasser M, De Caterina R. Hepatocyte growth factor: molecular biomarker and player in cardioprotection and cardiovascular regeneration. Thromb Haemost 2012; 107: 656-61.

23 Nakamura T, Mizuno S, Matsumoto K, Sawa Y, Matsuda H, Nakamura T. Myocardial protection from ischemia/reperfusion injury by endogenous and exogenous HGF. J Clin Invest 2000; 106: 1511-9.

24 Futamatsu H, Suzuki J, Mizuno S, Koga N, Adachi S, Kosuge H, et al. Hepatocyte growth factor ameliorates the progression of experimental autoimmune myocarditis: a potential role for induction of T helper 2 cytokines. Circ Res 2005; 96: 823-30.

25 Mizuno S, Kurosawa T, Matsumoto K, Mizuno-Horikawa Y, Okamoto M, Nakamura T. Hepatocyte growth factor prevents renal fibrosis and dysfunction in a mouse model of chronic renal disease. J Clin Invest 1998; 101: 1827-34.

26 Gong R, Rifai A, Dworkin LD. Anti-inflammatory effect of hepatocyte growth factor in chronic kidney disease: targeting the inflamed vascular endothelium. J Am Soc Nephrol 2006; 17: 2464-73.

27 Okunishi K, Dohi M, Fujio K, Nakagome K, Tabata Y, Okasora T, et al. Hepatocyte growth factor significantly suppresses collagen-induced arthritis in mice. J Immunol 2007; 179: 5504-13.

28 Mizuno S, Matsumoto K, Li MY, Nakamura T. HGF reduces advancing lungfibrosis in mice: a potential role for MMP-dependent myofibroblast apoptosis. FASEB J 2005; 19: 580-2.

29 Kuroiwa T, Kakishita E, Hamano T, Kataoka Y, Seto Y, Iwata N, et al. Hepatocyte growth factor ameliorates acute graft-versus-host disease and promotes hematopoietic function. J Clin Invest 2001; 107: 136573. 